\title{
Über das Verhalten und die Bedeutung des elastischen Fasernetzes in der Lunge bei Er- krankung an Tuberkulose nebst Betrachtungen über den Begriff der Disposition.
}

\author{
Von \\ [) Y. Karl Hess, \\ Jeitenfem Arat Aer Kurastalt Hainstein bei Fisenal.
}

Die verschiedenen histologischen Elemente der emzelnen Organe setzen schädigenden Nomenton, dio m einer Erkrankmg führen, cinen verschiedengradigen Widerstand entgegen. So verden z. B. in der Niere die arten Fithelion ciner Schädigung leichter unterliegen, als das stiitzende Bindegowebe orler gar die Kapsel; in Darm wird das Epithel dor Schleimhat und der Serosaiberzng einem Insult gegeniber weniger widerstandsfabig sein, als etwa die Muskularis. Im Respirationstraktus werlen wir in dieser beziehung einen erheblichen Untersched erwarten zwischon den zartun Githelien der Sohlemhant einerseits, dem Bindegewebe, elastischen Gewebe und dem Knorpel andererseits, welche chemischen, thermischen, mechanischen oder infektiösen Einflissen gegenüber sehr viel standiafter seir werden, als jene. Der Knorpel, der ja in der Lunge selbst normalerweise nicht mehr vorkommt, sondern nur in den Luft zuführenden Wegen. soll im nachColgenden gan\% ansser Betrach. bleiben, anch die Bathelien werden nur gelegentich beriicksichtigt.

In der Hanptsache sind die nachfolgenden Mitteilungen und Tntersuchungen dem elastischen Gewebe in der Lunge gewidmet, insbesondere seinem Verhalten bei infektiösen und destruierenden Prozessen, spexiell bei der Lungentiberkulose. Ein weiterer Abschnitt soll dann dartun, inwiefern die gewonnenen histologischen Resultate bei dem vielgestaltigen Begrift der "Disposition" eine guwisse Rolle zu spielen geeignet sind. 
I.

Aus den Untersuchungen von Lins e ${ }^{1}$ ) über den Bau und die Entwickelung des elastischen Gewebes in der Lunge wissen wir, dass sich dasselbe beim Menschen und wohl auch bei allen anderen Säugetieren erst nach der Geburt ausbildet. Was im embryonalen Leben davon vorhanden ist, kann nicht als voll angesehen werden, da es sich bei Färbungen auf elastische Fasern nur schwach färbt und damit als Vorstadium, als ,junges" elastisches Gewebe gekennzeichnet wird. Nach der Geburt erfolgt die Entwickelung des elastischen Netzes in der Lunge rasch, indem schon nach einem Monat es seinen späteren Stand fast vollkommen erreicht. $\mathrm{Zu}$ ähnlichen Resultaten kam Sudsuki²), der die elastischen Fasern der grösseren Gefässe zwar schon beim Fötus von $15 \mathrm{~cm}$ Kopf-Steisslänge voll ansgebildet fand, diejenigen der Alveolarwandungen aber noch gar nicht, während sie beim Fötus von $25 \mathrm{~cm}$ erst sehr schwach entwickelt waren. Bei Kindern von einem Monat waren sie dagegen bereits völlig ausgebildet.

Die physiologische Bedeutung der elastischen Lungenfasern, nicht nur für die Lunge selbst, sondern für den ganzen Organismus tritt uns deutlich vor Augen, wenn wir bedenken, dass sie es ermöglichen, dass die Alveole bei der Inspiration sich um das dreifache ihres Durchmessers erweitert und bei der Exspiration zu ihrem ursprünglichen Durchmesser von $0,1-0,3 \mathrm{~mm}$ wieder zurückkehrt. (Stöhr, Lehrbuch der Histologie.)

Daraus ergibt sich unmittelbar, dass sie direkt auf́ die Grösse der Lungenventilation bei der Atmungstätigkeit einen wesentlichen Einfluss haben müssen, indirekt sodann auf die Zirkulation und zwar zunächst im kleinen, in der Folge aber auch im grossen Kreislaufe. Pathologische Veränderungen stärkeren Grades im elastischen Fasernetze der Lunge werden also Störungen dieser beiden so wichtigen Funktionen des Organismus nach sich ziehen können.

Da die elastischen Fasern ausser ihrer Elastizität auch eine grosse Festigkeit besitzen, so werden sie dem Organ, das sie durchziehen und stuitzen auch eine grössere Widerstandsfähigkeit gegenüber von aussen wirkenden schädigenden Einflüssen verleihen. Die Lunge ist solchen Einwirkungen in nicht unerheblichem Masse ausgesetzt, und da sie im übrigen vorzugsweise aus zarten Epithelien und feinsten Gefässverzweigungen besteht, so dürfte dieser Punkt auch noch besondere Bedeutung erlangen.

Über die Anordnung der elastischen Fasern in der Lunge sagt

1) Anatom. Hefte, Bd. XIII, 1900.

2) Über Lungenemphysem, Virch ow s Archiv, Bd. 157, 1899. 
Stöhr ${ }^{1}$ ), dass die Wandungen der Alveolen und der Alveolengänge ausser den Muskelfasern der letzteren noch aus einer leicht streifigen Grundlage und vielen elastischen Fasern bestehen. Diese sind an den Alveolengängen zirkulär angeordnet. An der Basis der Alveolen bilden sie einen dicken Ring, während feine geschlängelte Fäserchen in ihrer ganzen Wandung vorkommen. Indem die elastischen Ringe benachbarter Alveolen an den Berührungspunkten miteinander verwachsen, bilden sie die Alveolensepta.

Sehr viele feine elastische Fasern finden sich ferner an der Pleura visceralis, während die Pleura parietalis ärmer daran ist.

Noch ausführlicher schildern diese wichtigen histologischen Verhältnisse Sudsuki und Linser. Letzterer sagt in seiner vorhin erwähnten Arbeit: „Die Alveolen sind bald von einer breiten Lage elastischer Fasern umgeben, bald nur von einer oder ein paar feinen Fibrillen, bald liegt die fibrilläre Grundsubstanz allein unter dem Epithel. Ein deutlicher Zusammenhang oder ein allmähliches Ineinanderübergehen der verschiedenen Formen besteht nicht. Die stärkste Entwickelung des elastischen Gewebes liegt immer in den Alveolarsepten vor, die gewissermassen aus der allgemeinen Wand des Endbläschens vorspringen. Die Fasern sind hier sehr dick und ziehen gegen die Spitze des Septums, indem sie zuerst konvergieren und darn wieder ganz wenig auseinanderweichen, um von vorn bedeckt zu werden durch den erwähnten Ring, der den Septumeingang in Gestalt mehrerer ziemlich dicker elastischer Fasern umgreift." Scheinbar planlos eingestreute Anschwellungen des elastischen Gewebes sind gelegentlich durch den Verlauf der Kapillaren bedingt, wie überhaupt vielfach die feinen Fasern in der Wand der Alveole dem Kapillarverlauf sich anschliessen, teils aber auch ein ganz unregelmässiges Netzwerk bilden. In dem elastischen Fasernetz der Gefässe und der Bronchiolen findet das Netzwerk der Alveolen gewissermassen einen Stützpunkt, indem von da aus gröbere und feinere Fasern nach allen Richtungen ausstrahlen.

Darin stimmen alle Autoren (Linser, Sudsuki, Tendeloo u. a.) überein, und auch meine Untersuchungen an der normalen Lunge führten mich zu dem gleichen Ergebnis, dass sich die elastischen Fasern in bezug auf ihre Reichlichkeit, Stärke und Anordnung bei verschiedenen Individuen ganz ausserordentlich verschieden verhalten, so dass sich eine bestimmte Regelmässigkeit nicht auffinden lässt. Diese Tatsache erscheint mir für eine Betrachtung im zweiten Abschnitt dieser Arbeit von wesentlicher Bedeutung, und ich möchte

1) Lehrbuch der Histologie, X. Aufl., 1903. 
als weiteren hierfür wichtigen Punkt gleich die Beobachtung anfügen, dass die elastischen Fasern, auch gegenüber der spezifischen Weigertschen Färbung, also in chemischer Beziehung, bei verschiedenen Individuen sich nicht gleichmässig verhalten. In einzelnen Fällen nehmen sie die Färbung leichter an als in anderen, trotz gleicher Vorbehandlung, und ebenso zeigt sich eine verschiedengradige Widerstandsfähigkeit der gefärbten Fasern gegenüber der nachfolgenden Alkoholbehandlung.

Die pathologischen Veränderungen, welche die elastischen Fasern in der Lunge am häufigsten erfahren, sind zweierlei Art: einmal der Verlust der Elastizität, wie er am charakteristischsten beim Emphysem zum Ausdruck kommt, sodann Verschiebungen, Lockerung ihres Gefüges und schliesslich Ausstossung oder völlige Zerstörung. Die makroskopischen Bilder hierfür liefern insbesondere der Abscess, die Gangrän und vor allem die Tuberkulose der Lungen in den verschiedenen Stadien ihres Verlaufes.

Besonders instruktiv in dieser Beziehung sind aber die mikroskopischen Präparate. Zu ihrer Darstellung gibt die Weigertsche $\left.^{1}\right)$ Färbung auf elastische Fasern die vollkommensten Bilder, vereinzelt habe ich auch die Tänzer-Unnasche ${ }^{2}$ ) Orcein-Methode in Anwendung gezogen.

Die Gewebstücke, aus denen die Schnitte angefertigt wurden, waren teils in Celloidin, teils in Paraffin eingebettet, teils wurde das Gefriermikrotom unter Benutzung flüssiger Kohlensäure verwendet.

Die vollkommensten Bilder ergab die Paraffinmethode, weil hierbei die Einbettungsmasse entfernt wird, während das im Präparat zurückbleibende Celloidin einen graublawen Ton behält, der namentlich bei dicken Schnitten, wie sie zur Beurteilung des Fasernetzes oft wünschenswert sind, störend werden kann. Beim Gefriermikrotom bleibt der maschenartige Aufban nicht so schön erhalten, sondern er erleidet leicht Verzerrungen und Ausfälle. Zur Gegenfärbung der Kerne wurde Borax-Karmin, Lithion-Karmin und vorzugsweise AlaunKarmin verwendet, für die Färbung der Grundsubstanz diente gelegentlich auch Eosin.

Will man sich einen guten Überblick über den Aufbau der elastischen Fasern um die Alveolen und Alveolengänge verschaffen, so dürfen die Schnitte nicht zu dünn sein. Schnitte von $30-40$ Mikren bei verschieden hoher Einstellung des Tubus betrachtet, sind

1) Über eine Methode zur Färbung elastischer Fasern. Zentralbl. f. allgem. Pathologie u. path. Anatomie, 1898, Nr. 8/9.

2) Encyklopädie der mikrosk. Technik von Ehrlich, Krause, Mosse, Rosin u. Weigert, 1903. 
hierfür besser als dünnere, die das Netzwerk in seiner Verzweigung nicht mehr so vollkommen erkenmen lassen. Bei den dicken Schnitten verzichtet man besser auf die Gegenfärbung, weil dadurch die feineren Fasern leicht verdeckt werden. Die matt graublaue Farbe des Grund= gewebes genügt zur Orientierung, oder man vergleicht an dïnneren Schnitten mit der erwähnten Kernfärbung.

Von pathologischen Fällen habe ich, ausser der akuten croupösen Pneumonie, chronische Erkrankungen untersucht. Zunächst das Emphysem, bei dem man a priori auffallende Veränderungen erwarten könnte, dann - die ersteren mehr zum Zweck der Vergleichung Fälle von brauner Induration, Lungencirrhose, Zementarbeiterlunge, ossifizierende Pneumonie, Bronchiektasie mit Bronchorrhöe und namentlich Tuberkulose verschiedenen Grades, von dem miliaren Tuberkel bis zur ausgesprochenen Phthise. Das erforderliche Material wurde mir im Heidelberger pathologischen Institut durch Herrn Geh.-Rat Arnold in liebenswürdiger Weise zur Verfügung gestellt.

Bezüglich des Emphysems möchte ich, ohne auf meine eigenen Untersuchungen weiter einzugehen, die diese Erkrankang nur in beschränktem Masse, soweit es für das Hauptthema erforderlich schien, berücksichtigten, nur bemerken, dass wir in den Arbeiten von Sudsuki ${ }^{1}$ ) und Tendeloo ${ }^{2}$ ) gründliche Beobachtungen über das Verhalten der elastischen Fasern hierbei besitzen. Im Anschluss an Sudsuki konnte dann $\mathrm{Hansemann}^{3}$ ) zu seinen interessanten Ergebnissen über die Entstehung des Lungenemphysems gelangen, in denen der primären, rein mechanischen Vergrösserung der zwischen den Alveolen befindlichen Stomata eine besondere Bedeutung und Wichtigkeit zugeschrieben wird.

Es sei hier wenigstens gestattet, die Schlussfolgerung Tendeloos, die sich im wesentlichen mit den Resultaten von Sudsuki deckt, in dieser Frage anzuführen: „Das elastische Fasergerüst gleich grosser Lungenbläschen zeigt in bezug auf Stärke und Reichlichkeit der Fasern Verschiedenheiten, welche durchaus regellos und unabhängig davon sind, ob diese Bläschen akut oder chronisch, substantiell oder vikariierend oder senil emphysematös oder endlich absichtlich mehr oder weniger vergrösserte (aufgeblasene) normale Lungenbläschen sind." -

Nach solchen Voruntersuchungen wendete ich mein Interesse vorzugsweise dem Verhalten und namentlich der Widerstandsfähigkeit des elastischen Fasernetzes in der Lunge bei der Erkrankung

1) 1. c.

2) Studien über die Ursachen der Lungenkrankheiten, Wiesbaden 1902.

3) Sitzungsbericht d. Berliner med. Gesellschaft. Berl. klin. Wochenschrift, 1899, Nr. 20. 
an Tuberkulose zu. Eine solche Untersuchung schien mir um so wünschenswerter als die wichtigste mir zugängliche Arbeit: „Über das Verhalten der elastischen Fasern in tuberkulösen Lungenherden" von $\mathrm{Schmaus}{ }^{1}$ ) bereits vor dem Bekanntwerden der Weigertschen Färbungsmethode veröffentlicht ist, der Autor also auf die weniger Vollkommenes leistende Orceinfärbung und die Kalilauge-Methode beschränkt war.

Meine Fragestellung war folgende: Gestattet der Befund an den elastischen Fasern bei verschiedenen Fällen und Stadien der Lungentuberkulose einen Rückschluss a uf ihre Widerstandsfähigkeitgegenüber dieser Krankheit?

Liess sich diese Frage bejahen, so gewann eine zweite Frage Berechtigung, nämlich: Könnte eine schwankende Wider. standsfähigkeit des elastischen Fasernetzes in der Lange gegenüber tuberkulöser Erkrankung im Verein mit der wechselnden individuellen Entwickelung desselben und unter Berücksichtigung seiner Ausbildung in der ersten Lebenszeit als Faktor angesehen werden, der bei der Frage derindividuellen angeborenen Dispositiongegenüber der Infektion durch den Tuberkelbacillus Beachtung verdient?

Beginnen wir zunächst mit der Untersuchung von miliaren Tuberkeln in der Lunge, so lässt sich hierbei in vielen Fällen infolge Erhaltung des elastischen Fasernetzes noch der alveoläre Bau der erkrankten Stelle erkennen, und zwar können selbst ganz feine Fasern noch wohl konserviert sein. An anderen Stellen ist dies nicht mehr der Fall; ja man kann gelegentlich schon in dem frühen Stadium der einfachen Rundzellenanhäufung eine auffallende Verminderung, selbst ein völliges Fehlen der elastischen Fasern beobachten. Interessant sind aber namentlich die wechselnden Zwischenstufen beim typischen Tuberkel mit zentraler Verkäsung und epitheloiden Randzellen. Bei dieser Neubildung kann selbst in anscheinend gleichem Stadium ihrer Entwickelung das einbezogene Fasernetz sich sehr verschieden rerhalten. Neben der bereits erwähnten Konservierung der alveolären Anordnung finden sich die mannigfachsten Übergänge bis zu ihrer völligen Zerstörung.

Zunächst verschwinden die feinsten Fasern oder sie werden aus ihrem Zusammenhange mit den stärkeren Fasern gelöst und liegen mehr oder weniger zusammengeballt innerhalb der Zone der epitheloiden Zellen oder der Verkäsung. Das gleiche Schicksal erleiden

1) Verhandlungen des Kongresses für innere Medizin, 1895. 
schliesslich auch die mittelstarken und starken Fasern und man findet sie innerhalb der verkästen Zone bäufig in derselben charakteristischen Anordnung, wie man sie bei der Untersuchung des Sputums oft sieht.

An wieder anderen Stellen sind aber die elastischen Fasern nicht aus ihrem Gefüge losgelöst, sondern sie erscheinen förmlich zur Seite geschoben durch die Neubildung des Tuberkels. Letzterer ist dann wie von einem Wall oder Ring von Fasern umgeben, und dieser Wall kann einen mehr oder weniger vollständigen Kreis oder Kreisabschnitt bilden. Stellenweise können aber an diesen einzelne Interalveolarleisten noch so vollkommen in Form und Lage sich erhalten, dass sie Vorsprünge ins Innere der Tuberkelmasse bilden.

Besonders zeigen sich die die Alveoleneingänge umspannenden Fasermassen widerstandsfähig. Man erkennt ihre, im Durschschnitt charakteristisch plattenförmige Anordnung mit einem daranhängenden längeren oder kürzeren Rest der Interalveolarwandfasern oft deutlich im Inneren der Käsemasse.

Sehr widerstandsfähig sind die Fasergruppen, die ihren Stützpunkt an oder in der Wand von Gefässen oder Bronchiolen haben. Gelegentlich habe ich sogar parallele, dicht aneinander gelagerte Züge elastischer Fasern quer durch einen Tuberkel verlaufen und beiderseits an der Peripherie in die Fasermassen eines in gleicher Richtung ziehenden Gefässes sich einfügen gesehen, also wohl den früheren Gefässverlauf an der Stelle des gefässlosen Tuberkels.

Man kann nun nicht sagen, dass die geschilderten Veränderungen der elastischen Fasern etwa an jüngeren Tuberkeln durchgehends noch geringfügiger seien, als an älteren, sie sind vielmehr äusserst variabel, ja unter Umständen in jungen Tuberkein viel ausgesprochener als in den älteren.

Konfluieren mehrere Tuberkel, so werden die sie umziehenden Gruppen von Randfasern allmählich aus ihrem Gefüge gelöst, doch auch insofern unregelmässig, als man gelegentlich zwischen kleinen Tuberkeln kaum noch einige Reste von Fasern finden kann, während andererseits beim Zusammenfliessen grosser Tuberkelmassen die exhaltenen Fasern lange durch ihre Zugrichtung die frühere Grenze der einzelnen Herde noch erkennen lassen können. Da auch hier das gilt, was vorher von der besonderen Widerstandsfähigkeit der Fasern an und in den Gefässen und Bronchiolen gesagt wurde, so ist es begreiflich, dass man selbst bei ausgedehnteren käsigen Einschmelzungen Anordnungen von Fasern trifft, die als Reste von Gefässen und grösseren oder kleineren Bronchiolen anzusprechen sind. Doch erhalten sich auch nicht selten Fasern in der charakteristischen Anordnung der Alveolen lange Zeit inmitten einer grösseren Käsemasse. 
Erfolgt ein Durchbruch solcher Herde nach aussen und ihre Entleerung durch das Sputum, so wird man sich nicht wundern, darin diese verschiedensten Anordnungen der elastischen Fasern in mehr oder weniger vollkommener Erhaltung zu finden. Am häufigsten ist die Anordnung der zusammengeschobenen interalveolaren und der interlobulären Leisten. -

Selbst bei stark destruierenden Formen der Phthise kann der alveolare Bau des Fasernetzes noch relativ lange kenntlich bleiben oder die Verschiebungen und Umlagerungen erfahren, wie sie eben geschildert wurden. An anderen Stellen kann aber das elastische Gewebe völlig verschwunden oder wenigstens nur in grösseren oder kleineren zerstreuten oder zusammengeballten Trümmern in den käsigen Massen nachweisbar sein.

Unter Berücksichtigung der verschiedenen Bilder, die sich ergeben müssen, je nach dem ursprünglichen Sitz des tuberkulösen Herdes, sei er intra- oder interalveolär, sei er bronchial oder peribronchial, oder in den Gefässscheiden, glaube ich, dass man berechtigt ist, zur Erklärung der geschilderten, überaus wechselnden und schwankenden Widerstandsfähigkeit gegenüber der aufösenden Wirkung des tuberkulösen Prozesses spezifische Eigentümlickeiten des elastischen Fasernetzes heranzuziehen, mögen dieselben nun in noch unbekannten Bedingungen chemischer Natur beruhen, oder auf den tatsächlich vorhandenen individuellen Schwankungen in der Mächtigkeit und Entwickelung der einzelnen Fasern und des Gesamtfasernetzes.

II.

Nachdem somit die erste der gestellten Fragen sich in bejahendem sinne beantworten liess, gestattet sie meines Erachtens eine Schlussfolgerung, die für die Beurteilung des Zustandekommens und der Ausbreitung von manchen Infektionskrankheiten in der Lunge und speziell der Lungentuberkulose von einer gewissen Bedeutung ist.

Indem ich zur Darlegung dieser Folgerung übergehe, muss ich zunächst in Kürze meine Auffassung über die Entstehungsbedingungen der Lungentuberkulose aussprechen, wie ich sie mir in langjähriger klinischer Beobachtung während meiner Tätigkeit in der Heilanstalt Falkenstein i. T. gebildet habe. Mutatis mutandis dürfte das Anzuführende auch für gewisse andere Infektionsarten Geltung beanspruchen.

Zum Zustandekommen einer manifesten Erkrankung an Lungentuberkulose sind zwei Dinge erforderlich: einmal die Infektion mit dem pathogenen spezifischen Krankheitserreger und sodann die Disposition des von ihm befallenen Organs oder Individuums. 
9] Über das Verhalten u. die Bedeutung des elastischen Fasernetzes etc. 111

Beide sind gleichermassen dazu erforderlich, insofern als das einzelne Moment an sich die Krankheit noch nicht ohne weiteres auslöst. Der Streit, welches von beiden das "Wichtigere" sei, erscheint mir müssig; sie sind eben beide erforderlich, sie müssen sich im Organismus begegnen. Sie stehen aber in gewissen Wechselbeziehungen zueinander, über die wir bis jetzt nur beschränkte Kenntnisse haben, und deren Klärung ein zwar schwieriges, aber dankbares Kapitel der wissenschaftlichen Forschung bildet.

Infektionserreger und Disposition können verschiedene Intensitätsgrade ihrer Wirkung aufweisen, die in ihrer Wechselbeziehung die Schwere der Erkrankung bedingen.

Die Virulenz der ersteren beim Eindringen in den Organismus kann aus verschiedenen Gründen verschieden hochgradig sein. Zunächst hängt sie $a b$ von der aus der ursprünglichen Quelle mitgebrachten Virulenz, sodann von etwaigen, während des Aufenthalts ausserhalb des menschlichen Körpers günstig oder ungünstig auf den Bacillus wirkenden Faktoren. Dabei kommen in Betracht Austrocknung oder Feuchtigkeit, Besonnung oder Beschattung, etwaiges Auffallen auf einen seiner Erhaltung mehr oder weniger günstigen Boden, kurz Faktoren, die wir ziemlich genau kennen und die wir auch zum Teil im Laboratorium experimentell studieren können.

Bei manchen Krankheitserregern, z. B. dem der Cholera und des Typhus finden solche Faktoren gewissermassen einen Ausdruck in besseren oder schlechteren hygienischen Einrichtungen und Bedingungen, bei anderen in klimatischen Verhältnissen, wie beim Influenzabacillus und anderen, die bei feuchtem nebligen Wetter relativ widerstandsfähig sind, bei Austrocknung aber rasch zu grunde gehen.

Auch vom Tuberkelbacillus wissen wir, dass seine Virulenz von vornherein in verschiedenen Krankheitsfällen ausserordentlich verschieden ist, dass wir vom ganz schwach virulenten bis zum Erreger schwerster Infektion mancherlei Zwischenstufen beobachten können. Wir wissen ferner, dass er sich ausserhalb des menschlichen Körpers lange lebensfähig und infektionskräftig erhalten kann, und dass er diese Fähigkeiten am längsten und stärksten in geschlossenen etwas dumpfen Räumen behält, während er gegen Luft und Licht empfindlich ist und durch strahlendes Sonnenlicht sehr bald abgetötet wird. Bezüglich seiner Dauerhaftigkeit im Staub geschlossener Räume brauche ich nur an die bekannten Untersuchungen von Cornet zu erinnern, ja unter besonders günstigen Bedingungen kann er sich sogar ausserhalb des Körpers im ausgeworfenen Sputum vorübergehend vermehren, wie die Untersuchungen meines früheren Assi- 
stenten Dr. Gäthgens $\left.{ }^{1}\right)$ gelegentlich der Nachprüfung der Hesseschen Anreicherungsverfahren gezeigt haben.

Vorbedingungen hierfür sind ziemlich hohe Temperatur und genügend feuchte Luft, Bedingungen, wie sie gelegentlich in geschlossenen Räumen, z. B. einer Fabrik oder in Schiffen vorkommen dürften.

Hess $\left.e^{2}\right)$ selbst hat die Möglichkeit der Vermehrung der Tuberkelbacillen im ausgeworfenen Sputum kürzlich bestätigt. -

Während unsere Kenntnisse über die Biologie des Tuberkelbacillus durch zahlreiche Untersuchungen fortschreitend gefördert wurden, verbirgt sich hinter dem Begriff "Disposition" noch eine überaus grosse Menge von Unklarheit. $\mathrm{Er}$ ist so vielgestaltig und umfasst so viele geklärte und ungeklärte differente Faktoren, dass ich der Übersicht halber zunächst eine Gruppierung derselben vornehmen möchte. Fine solche kann von verschiedenen Gesichtspunkten aus erfolgen, und man muss sich dabei bewusst bleiben, dass es sich nicht immer um scharfe Abgrenzungen handeln kann, dass vielmehr einzelne Glieder gelegentlich ineinander iibergehen oder je nach der Auffassung auch zu einer anderen Gruppe gezählt werden können. Ein starres Schema ist in solchen Dingen ja unmöglich.

Seit langem und zweckmässig unterscheiden wir bekanntlich zwischen angeborener und erworbener Disposition. Bei letzterer kann man noch eine chronische, d. h. auf lange Zeit, eventuell auf Lebensdaner akquirierte Form trennen von einer temporären, die rasch im Anschluss an andere Krankheiten entsteht und wieder verschwindet, wie z. B. die Disposition zu Tuberkulose nach Influenza oder nach Masern. Von anderen Gesichtspunkten unterscheiden wir auch eine lokale und eine allgemeine Disposition.

Insgesamt beruht sie auf vorwiegend anatomisch oder vorwiegend physiologisch-chemisch oder physikalisch aufzufassenden Schwankungen in dem Aufbau des Organismus. Auch nach diesem Gesichtspunkte lässt sich demnach gruppieren, wie in nachfolgendem geschehen soll.

Dabei kann vorerst ausser Betracht bleiben, ob man sich der Auffassung der aërogenen, der hämatogenen oder der lymphogenen Kntstehung der Lungentuberkulose anschliesst.

Ich will die einzelnen Glieder der Gruppen nicht alle erschöpfend aufzählen und besprechen. Es würde dadurch der Rahmen dieser Arbeit überschritten, und ich behalte mir vor; vielleicht bei anderer

1) Zeitschrift f. Tuberkulose u. Heilstättenwesen, 1900.

2) Deutsches Archiv für klin. Medizin, Bd. 77, 1908. 
11] Über das Verhalten $u$. die Bedeutung des elastischen Fasernetzes etc. 113

Gelegenheit ausführlicher darauf zurïckzukommen. Die Gruppen seien nur umgrenzt: und ihre charakteristischsten Glieder angeführt.

Die vorwiegend anatomisch sich darstellenden Dispositionsmomentekönnen makroskopisch odermikroskopisch sichtbar sein.

Makroskopisch hervortretende finden wir am knöchernen Ger üst des Thorax in dem Verhalten des Sternums, speziell des Sternalwinkels, auf dessen Bedeutung Rothschild $\left.{ }^{1}\right)$ hingewiesen hat, sowie in der Beschaffenheit der ersten Rippe, die Freund ${ }^{2}$ ) nenerdings wieder $z u$ eingehenden Untersuchungen und sogar zum Vorschlag der operativen Durchtrennung dieser Rippe hart am Manubrium sterni veranlasst hat, im Hinblick auf die Beobachtung der heilenden Wirkung einer Gelenkverbindung an dieser Stelle.

Auch die von $\mathrm{Schmorl}^{3}$ ) beschriebene disponierende Furchenbildung, 1-2 cm unterhalb der Lungenspitze ist auf die mangelhafte Entwickelung der ersten Rippe, ihr abnorm weites Vorspringen in die Thoraxkuppel zurückzuführen. Dadurch könnten wieder die weiter unten noch zu erwähnenden Verkümmerungen des hinteren Spitzenbronchus, Ventilations- und 7irkulationsstörungen, Sekretstauungen, Katarrhe u. s. w. begünstigt werden.

Weiter sind hier die Difformitäten anzugliedern, welche der Thorax durch Wirbelerkrankungen wie Kyphose und Skoliose erleiden kann, dann die Trichter- und Hühnerbrust, sowie andere durch Traumen gesetzte Verunstaltungen.

Die schlechthin als Habitus phthisicus und paralytischer Thorax bezeichnete Formveränderung ist in der Regel die Folge des Zusammentreffens mehrerer dieser genannten Faktoren. Sie scheint übrigens in den Lehrbüchern und in der ärztlichen Praxis im Vergleich zu anderen disponierenden Momenten immer noch allzu sehr betont zu werden.

$\mathrm{Zu}$ den makroskopisch schon nachweislichen disponierenden pathologischen Veränderungen in den Weichteilen sind die

1) Der Sternalwinkel in anatomischer, phys. und path. Hinsicht. Frankfurt a. M., 1900 .

2) Thoraxanomalien als Prädisposition zu Lungenphthise und Emphysem. Berl. klin. Wochenschr. 1902, Nr. 1 u. 2. - An dieser Stelle sei auch auf die für die Frage der Disposition bedeutungsvolle Diskussion über Freunds Vortrag in der Medizin. Gesellschaft zu Berlin hingewiesen, insbesondere auf die Erörterungen von A. Fraenkel und Liebreich, ebenda Nr. 2-4. - Vergl. ferner: Rothschild, Die Funktionen der ersten Rippe. Verhandl. d. Kongr. für innere Medizin. Wieshaden 1902.

3) Beginn der Lungentuberkinlose. Nünch. med. Wochenschr. 1901. 
Folgen sich häufig wiederholender akuter, sowie der chronischen Katarrhe und Entzündungen der Bronchien und Lungen, und ferner die cirrhotischen Prozesse zu rechnen. Ebenso gehören hierzu die zu Schleimhautatrophie führenden Nasen- und Kehlkopfkatarrhe, z. B. der Cigarrenarbeiter, deren Bedeutung besonders Brauer ${ }^{1}$ ) gewürdigt hat.

Auch die Verkümmerung der Entwickelung gewisser Bronchialäste im Gebiete des Bronchus apicalis posterior, worüber wir BirchHirschfel $d^{2}$ ) interessante Untersuchungen verdanken, sind hier anzuführen.

Dass ferner mehr oder weniger ausgedehnte pleuritische Verwachsungen durch Beschränkung des benachbarten Lungengewebes in der Respiration disponierend zu wirken vermögen, ist wohl nicht zu bezweifeln. Allerdings ist hierbei zu berücksichtigen, dass sehr viele Fälle von Pleuritis erst sekundär, infolge primärer Lungentuberkulose entstehen. Es kommen also hier natürlich nur die pleuritischen Verwachsungen in Betracht, die nicht primär tuberkulös sind.

Endlich sei an dieser Stelle noch auf die angeborene Pulmonalstenose in ihrer Bedeutung für die Entstehung der Lungentuberkulose hingewiesen. -

$\mathrm{Zu}$ den lediglich oder vorzugsweise der mikroskopischen Untersuchung zugänglichen disponierenden Faktoren gehören geringgradige katarrhalische und sonstige entzündliche Prozesse des epithelialen Überzugs des Respirationstraktus, welche die Vitalität der Zellen schwächen und dadurch dem infizierenden Virus den Eintritt in den Organismus erleichtern, ohne deswegen sogleich zu groben anatomischen Veränderungen zu führen.

In diese Gruppe möchte ich auch die verminderte Widerstandsfähigkeit des elastischen Fasernetzes gegenüber dem tuberkulösen Prozess rechnen. Sie ist als eine angeborene Disposition zu bezeichnen. Verschiedene Punkte verdienen dabei Berücksichtigung. Zunächst kann man sich vorstellen, dass die individuell verschieden starke Entwickelung und Mächtigkeit des Fasernetzes direkt einen entsprechend verschiedenen Widerstand der Entwickelung der ersten pathologischen Neubildung des Tuberkels entgegensetzt, und dass das Fortschreiten des tuberkulösen Prozesses, insbesondere der tuberkulöse Zerfall darin ein individuell verschiedenes Hemmnis findet. - Der Umstand, dass die elastischen Fasern die

1) Beiträge zur Klinik der Tuberkulose I, 1902.

2) Dentsch. Archiv f. klin. Medizin, Bd. 64 und Bericht über den Kongress zur Bekämpfung der 'luberkulose als Volkskrankheit. Berlin 1899. 
spezifische Färbung in verschiedenen Fällen ungleich schnell annehmen und sich auch wieder individuell verschieden gegenüber der Alkoholbehandlung in der Farbabgabe verhalten können, lässt an weitere Schwankungen in der Beschaffenheit des Elastins denken.

Die Tatsache, dass das jugendliche Alter so sehr durch die Tuberkulose gefährdet ist, steht möglicherweise auch in einem gewissen Zusammenhang mit der später, erst post partum, sich vollendenden Entwickelung des elastischen Fasernetzes in der Lunge. Diese wie manche andere Frage bedarf indes noch weiterer Durchforschung, wozu mir die Zeit und ein entsprechendes Material fehlten.

Unter anderem scheint mir der Bearbeitung wert, ob nicht in den Fällen, in welchen ganze Familien und Generationen hintereinander der Tuberkulose erliegen - Vorgänge, über deren Grundursache wir noch berzlich wenig wissen - auch familiäre Eigentümlichkeiten in dem histologischen Aufbau der Lungen, speziell ihres Fasernetzes nachweisbar sind.

Indirekt hat aber jedenfalls die Beschaffenheit des letzteren einen begünstigenden oder hemmenden Einfluss auf das Zustandekommen von Erkrankungen, nicht allein tuberkulöser Natur, durch seine Bedeutung für die Zirkulation in der Lunge. Ihre inspiratorische Dehnung und deren Einfluss auf die Weite der Gefässe ist in hohem Masse von der Verfassung der elastischen Fasern abhängig. Besonders müssen hier die durch diese gebildeten Verbindungen zwischen den Gefässen und den Alveolarwandungen von Wichtigkeit sein, worauf auch Esser ${ }^{1}$ ) in seinem „Beitrag zur Lehre von der Störung der Lungenzirkulation" hingewiesen hat. -

Sehr ausgedehnt, aber noch wenig geklärt ist das Gebiet der Disposition auf chemischer Grundlage. Hierher gehört die auffallende Neigung zur Erkrankung an Tuberkulose nach gewissen akuten Krankheiten wie Masern, Keuchhusten und Influenza. Auch von einzelnen chronischen Krankheiten müssen wir annehmen, dass sie Änderungen im Stoffwechsel herbeiführen, die für das Haften und die Weiterentwickelung der Krankheitskeime günstig sind, so von hartnäckigen Anämieen und Chlorosen, dem chronischen Alkoholismus und vor allem dem Diabetes.

Nicht genügend beachtet in der Praxis scheint mir die Bedeutung der chronischen Influenza für die Begünstigung der Entstehung oder das Progressivwerden einer bereits vorhandenen Tuber-

1) Zentralbỉ, für innere Medizin 1901, Nr. 4. 
kulose. Es ist das Verdienst von Finkler ${ }^{1}$ ) hierauf besonders hingewiesen zu haben.

Die grössere Gefahr, die eine tuberkulöse Infektion während der Pubertät bringt, und die Neigung der Krankheit zu rapiderem Verlauf während des Puerperiums gehört ebenfalls in dieses Kapitel.

Rein mechanisch können auch Hindernisse in den oberen Luftwegen, wie Erkrankungen in der Nase und Wucherungen im Nasenrachenraum, die Atmung erschweren und somit die genügende Sauerstoffzufuhr einschränken.

Die Tatsache, dass Überarbeitung, Kummer und Sorgen zur Erkrankung zu disponieren vermögen, kann nur ihre Erklärung finden in Störungen, die die normalen chemischen Vorgänge im Körper erleiden.

Von grossem Interesse sind in diesem Zusammenhange endlich die von Hesse kürzlich mitgeteilten Beobachtungen in der schon vorher kurz erwähnten Arbeit ${ }^{2}$ ), wonach es gelingt, in jedem Auswurfe die in ihm enthaltenen Tuberkelbácillen zu weiterem Wachstum zu bringen, wenn man nur dem Nährboden den geeigneten Alkalescenzgrad verleiht. Die ausgeworfenen oder beim Husten verspritzten Tuberkelbacillen befinden sich in Schleimmengen, welche die für die betreffende Kolonie günstigste Alkalescenz besitzen. Kommen nun bei der Übertragung durch die Flüggeschen Tröpfchen oder durch Staub die Tuberkelbacillen bei einem gesunden Menschen in stockenden Bronchialschleim, der dieselbe oder nahezu dieselbe, also für die betreffende Kolonie optimale Alkalescenz besitzt, so werden sie sich schon innerhalb weniger Stunden, und ehe der Schleim nach aussen geschafft werden konnte, darin vermehren und zur Entwickelung der Krankheit Veranlassung geben können. Daraus erklärt es sich auch teilweise, warum die Lungentuberkulose so häufig an Stellen beginnt, an welchen am leichtesten eine Stockung von Bronchialschleim statt hat. -

Es würde zu weit führen, hier ausführlich auf alle diese Fragen einzugehen $^{3}$ ). Die chemischen Vorgänge im Organismus, welche denselben gegen Infektionen anfällig machen oder gegen solche schützen; sind jedenfalls sehr kompliziert und uns vielfach noch unbekannt. Sie

1) Infektionen der Lunge durch Streptokokken und Influenzabacillen. Bonn 1895.

2) Über die Bedeutung des Auswurfs als Nährboden für den Tuberkelbacillus. Deutsch. Archiv für klin. Medizin, Bd. 77, Heft 5/6, 1903.

3) Siehe hierzu auch: Martius, Pathogenese innerer Krankheiten. Leipzig und Wien 1899. 
15] Über das Verhalten u. die Bedeutung des elastischen Fasernetzes etc. 117

sind aber von ausserordentlicher praktischer Wichtigkeit, denn die ganze Immunitätslehre, an deren Ausbau jetzt unsere tüchtigsten Forscher ihre Kräfte einsetzen, hängt eng mit ihnen zusammen. Von der Immunitätslehre und den aus ihr gezogenen praktischen Folgerungen erwarten wir heute das langersehnte spezifische Heilmittel gegen die beimtückische Tuberkulose, und die neuesten Arbeiten von $\mathrm{Koch}$ und von v. Behring und ihren Schülern auf diesem Gebiet lassen die Erreichung dieses hohen Zieles erhoffen. 